\title{
Applications of 2D/3D TOF-SIMS with Fast MS/MS Imaging and keV-CID Identification for Research and Industrial Problem Solving: Low-abundance Molecules, Stereoisomers, Monolayers \& Devices
}

\author{
Gregory Fisher
}

Physical Electronics, Chanhassen, Minnesota, United States

The manufacture of high-performance fibers is studied extensively to provide or to enhance specific characteristics including durability, stain resistance, print adhesion and metallization, breathability, moisture wicking, mildew resistance, heat resistance and flame retarding, to name a few. However, in the pursuit of designer chemistries for use in engineered fabrics, researchers must be able to ascertain the chemical and structural modifications caused by various treatment methods. Here, we evaluate the extent of 2D and 3D (pyro)phosphorylation of $100 \mu \mathrm{m}$-diameter cellulose fibers by thermal and plasma treatment. The TOF-SIMS tandem MS imaging analyses were conducted on a PHI nanoTOF II Parallel Imaging MS/MS instrument which was equipped with an Ar gas cluster ion beam (GCIB) for analysis in depth. A significant and crucial finding, observed in the keV-CID product ion spectra, is that the plasma treatment leads predominantly to phosphate reaction at primary alcohol groups of the cellulose whereas the thermal treatment is indiscriminate and leads to phosphate reaction at both primary and secondary alcohol groups. In general, the plasma treated samples had greater and more uniform phosphate and pyrophosphate content (Figures $1 \& 2$ ). The thermal treated samples were more inhomogeneous, and in some cases $1-5 \mu \mathrm{m}$-diameter phosphate "blisters" were observed.

TOF-SIMS imaging with parallel tandem MS imaging allows rapid and virtually non-destructive identification and visualization of molecular chemistry at unprecedented spatial resolution. The TOF-TOF mass spectrometer allows TOF-SIMS $\left(\mathrm{MS}^{1}\right)$ imaging and product ion $\left(\mathrm{MS}^{2}\right)$ imaging to be achieved simultaneously and in parallel [1]. Ions for parallel $\mathrm{MS}^{1}$ and $\mathrm{MS}^{2}$ imaging are produced by a primary ion nanoprobe wherein the data is collected in minutes. Fragmentation of molecular precursor ions within a monoisotopic window is accomplished by kiloelectron volt collision-induced dissociation (keV-CID). Lateral resolutions produced in both $\mathrm{MS}^{1}$ and $\mathrm{MS}^{2}$ images are demonstrated to be in the range of $70 \mathrm{~nm}$ to $1 \mu \mathrm{m}$. Whether in discovery research, failure analysis or reverse engineering, the objective is to ascertain the 2D/3D composition and the structure of molecules. Most often there is neither sufficient background information nor reference spectra for substantiation of the analytical observations. Hence, a tool must be employed which can add the capability of unambiguous identification together with detection of molecules at low abundance and high spatial resolution.

Other unique examples will also be highlighted. In one case, a carbon residue was observed by Auger electron spectroscopy (AES) imaging of etched and $\mathrm{e}^{-}$beam-patterned structures, but the source of carbon was indeterminate. In a second case, molecules having organic ligands were patterned by a photolithography method and ostensibly loaded with catalytically active metals. Spatially resolved mass spectrometry had been performed previously, but fell short of verifying the presence and structure of any metal-organic structures because there was no capability for structural differentiation of the detected ions. Differentiation and identification of structural isomers will also be discussed in an example of a contaminated medical device. Finally, the identification and imaging of low-abundance $(<20 \mathrm{ppm}$ abundance) molecules will be discussed in a spatiotemporal dynamics study of bioactive lipids in arteries undergoing restenosis. 


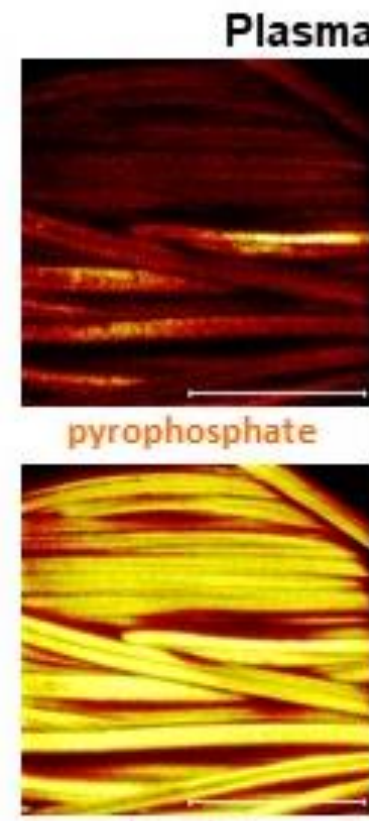

phosphate

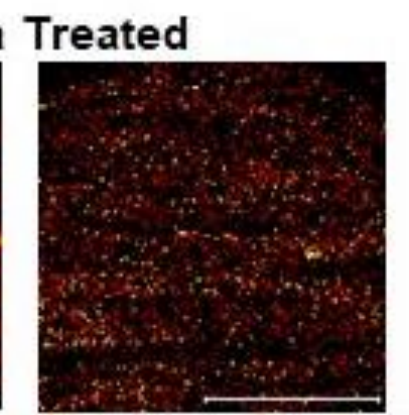

cellulose pyrophosphate

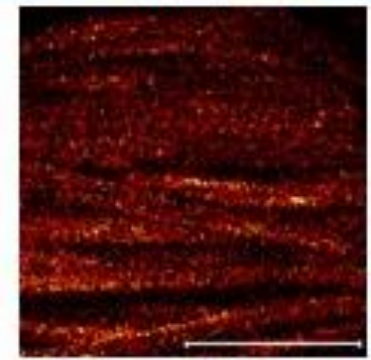

cellulose phosphate

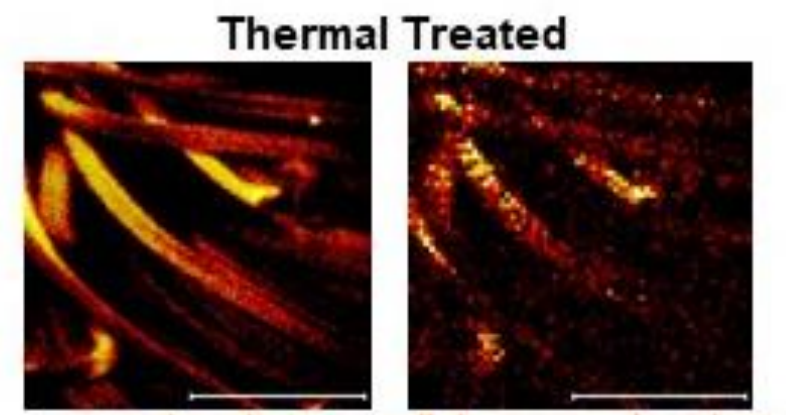

pyrophosphate

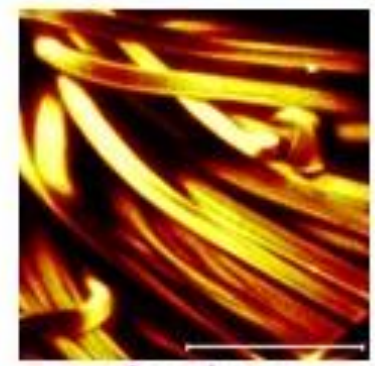

phosphate cellulose pyrophosphate

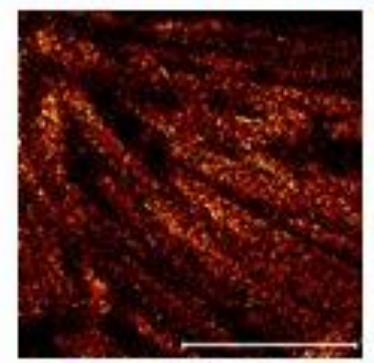

cellulose phosphate

Figure 1. TOF-SIMS tandem MS ion images in a $200 \mu \mathrm{m}$ x $200 \mu \mathrm{m}$ area of the plasma-treated (LEFT) and the thermal-treated (RIGHT) cellulose fiber samples. The primary ion fluence was equivalent in both sets of data. Specific phosphate moieties are shown in the bottom row, and specific pyrophosphate moieties are shown in the top row. The plasma treated samples had greater and more uniform (pyro)phosphate content, while the thermal treated samples were more inhomogeneous.
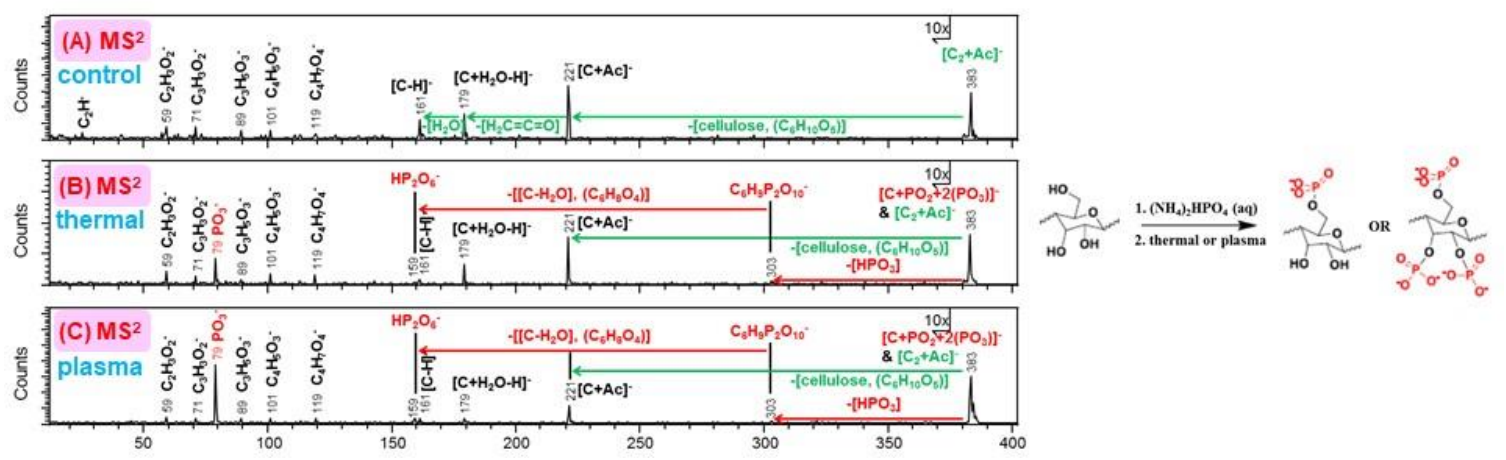

Figure 2. (LEFT) TOF-SIMS tandem MS spectra of the $\mathrm{m} / \mathrm{z} 383$ precursors from (A) the untreated cellulose fibers, (B) the thermal-treated cellulose fibers, and (C) the plasma-treated cellulose fiber samples. (RIGHT) A schematic of the idealized reaction scheme.

References

[1] (a) G.L. Fisher, et al, Anal. Chem. 88 (2016) 6433. (b) G.L. Fisher, et al, Microscop. Microanal. 23 (2017) 843. (c) C.E. Chini, et al, Biointerphases 13 (2018) 03B409. (d) T. Fu, et al, Nature Sci. Rep. 9 (2018) 1928. 